\title{
Design of Inventory Management Module in Printing Enterprise Based on Web
}

\author{
Peipei Ran ${ }^{1, \text { a }}$, Wenjie Yang ${ }^{1}$, Shaozhong $\mathrm{Cao}^{2}$ and Yuke Huo ${ }^{1}$ \\ ${ }^{1}$ School of Printing and Packing Engineering, Beijing Institute of Graphic Communication \\ ${ }^{2}$ Beijing Key Laboratory of Signal and Information Processing for High-End Printing Equipment, Beijing Institute of Graphic \\ Communication
}

\begin{abstract}
Based on the research and analysis of the management method for materials and combined the business process of material management, the inventory management module is designed and realized, which adopts the development mode of browser and server $(\mathrm{B} / \mathrm{S})$, and achieves the material warehousing, inventory, monthly closing functions. The implementation of module can effectively reduce inventory costs, optimize the management method and improve the economic efficiency
\end{abstract}

\section{INTRODUCTION}

Inventory management is a basic enterprise production and management activity, scientific management can systematically coordinate all departments and produce objective economic benefits [1]. With the change of customer requirements, the business of printing enterprise gradually develops in the direction of multivarieties and small batch. Therefore, the categories and quantities of materials in the enterprise's inventory are also increasing, and traditional manual or semi-manual recording methods can no longer adapt to the explosive growth of inventory information. Only realize the target of inventory management informationization, the requirements appeared in enterprise development can be met.

In order to solve the problems existed in the traditional inventory management, we combine the requirements of enterprises to implement management information, design and realize the inventory management module, which achieve the material warehousing, inventory, monthly closing and other functions, and the inventory management efficiency is effectively improved.

\section{Demand analysis}

Inventory management is an important part of enterprise management. In the business activities of a company, it is necessary to meet the needs of the workshop for the materials, but also to provide the procurement department with the inventory information in time, and to reduce the material inventory rate and waste without affecting production. In order to meet this requirement, the inventory department needs to work closely with the production and the purchasing department to update the material storage information in time.
The functions of the inventory management module designed in this paper are as follows.

(1) The entry management for material is realized, and the information from purchasing inventory or warehouse adjustment is input and submitted to be checked for manager. After checked, the stock in receipt is generated.

(2) The outbound management for material is realized, and the addition of the outgoing information according to the work order is completed, maintained or checked, and the stock out receipt is generated.

(3) The inventory management for material is realized, and the material is examined and counted regularly, after the actual quantity of the material and the number of the records of the account is checked, the result is input into the inventory sheet, the maintenance and audit of the inventory information is completed.

(4) The monthly management for materials is realized, and the warehousing information of the previous month at the beginning of each month is summarized to generate monthly receipt.

This paper introduces UML use case on the system function analysis, shown in Figure 1.

\section{System design}

\subsection{Overall design}

The entire process of material inventory management includes material warehousing, inventory and monthly closing. The data flow diagram is shown in Figure 2. 


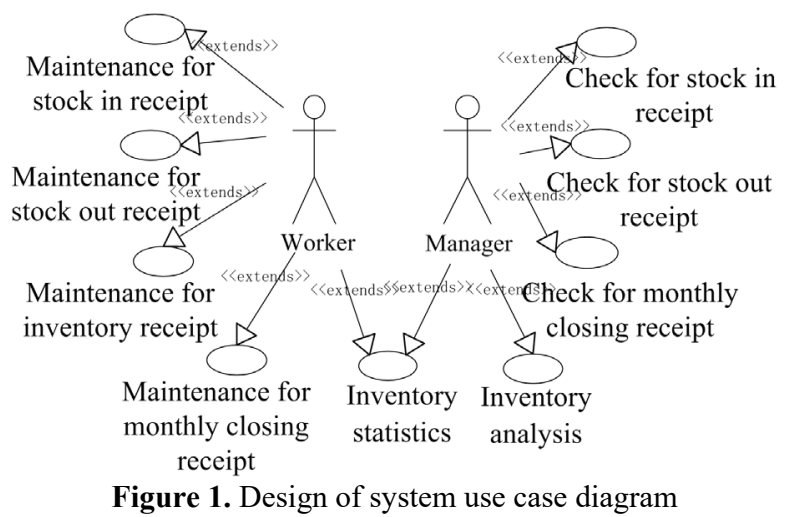

\subsection{Design of functions}

The main purpose of the inventory management module development is to realize the systematic and standardized management of material information, thereby improving the inventory management efficiency. Based on system requirements analysis and overall design, four sub-modules are contained in the inventory management module, which respectively achieve the material warehousing, inventory, monthly closing. The functions that each module needs to be implemented are as follows.

(1) All kinds of information from inventory management is input, including information from material warehousing, inventory and monthly closing.

(2) All kinds of information from inventory management is queried and modified.

(3) After added, all kinds of information from inventory management is submitted and checked.

(4) The inventory information of all materials is queried.

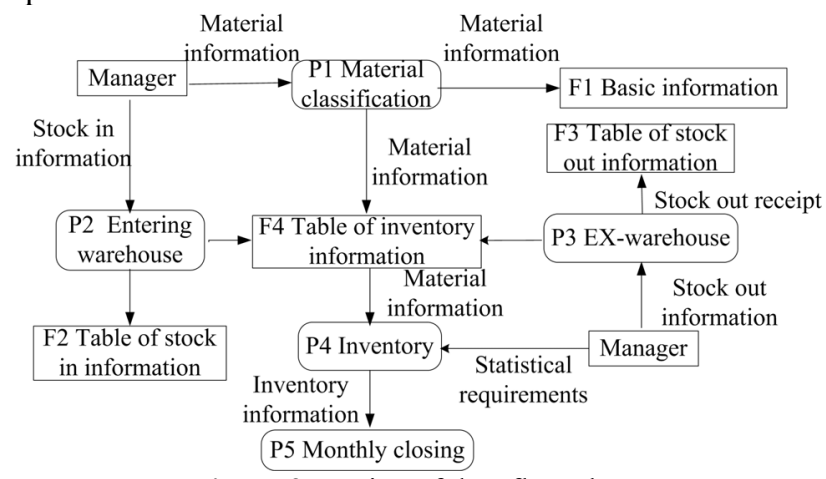

Figure 2. Design of data flow chart

\subsection{Design of database}

The design of structured database is a prerequisite for the data management effectively and a guarantee for generating correct information, and it is also the key to design management information system. Any system contains large amounts of data, when the database is properly designed, it will make the system more stable and efficient [3]. Combining demand analysis and system functions design, eight database tables are required in the inventory management module, they are respectively the tables of stock in receipt, stock in information, stock out receipt, stock out information, inventory receipt, inventory information, monthly closing information and stock information. Every two tables included in warehousing and inventory are linked by a keyword. The changes in the warehousing information of each materials are reflected in the warehousing tables, and represented in the inventory information table. When the material information is to settle accounts at the beginning of each month, the information stored in stock in and stock out tables are generated and calculated, and the result will be added to the monthly closing table for preservation.

The logical structure between the main database tables is represented by the E-R diagram, as shown in Figure 3 .

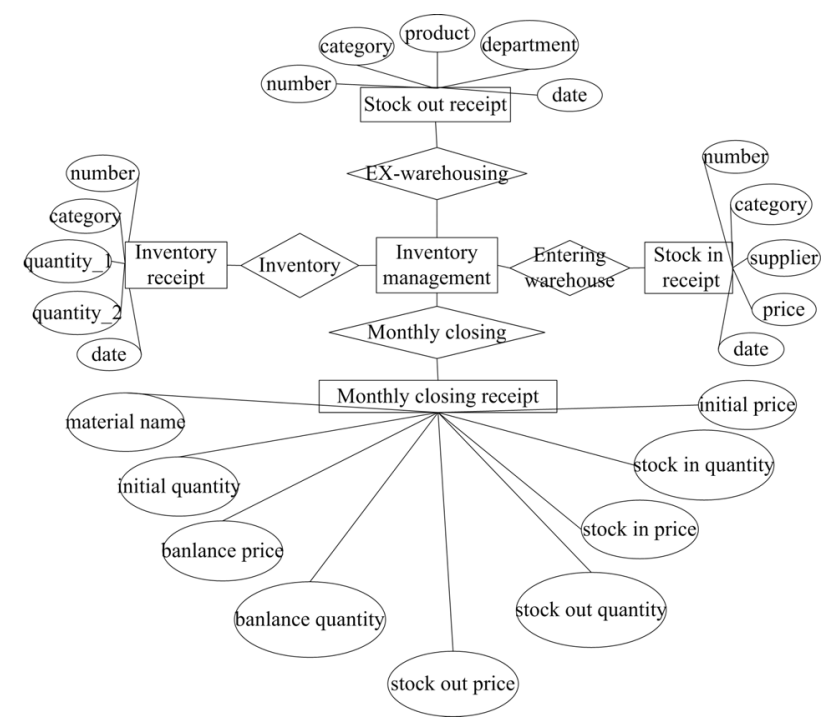

Figure 3. Design of E-R diagram

\section{System implementation}

\subsection{System implementation environment and technology}

Three-tier structure of browser/server $(\mathrm{B} / \mathrm{S})$ is used in system development, including the interface layer, application layer and data layer according to the difference of system functions, which are supported by the client, application server and data server respectively [4]. In the system, the business information is obtained through the interface layer, the network transmission between services are realized in the application layer, and the data information is managed according to relevant instructions in the data layer.

During the development process, the inventory management module is developed on the Eclipse platform using the Java language, and the software can be implemented on different platforms such as Windows, UNIX and Linux. The data information is managed in MySQL, which is a small relational database management system and the data is stored in different tables. SQL language, used to access the database, is the most common table conversion language, which is widely applied in development of the small and medium- 
sized website due to its small size, fast speed and low cost [5].

\subsection{Business process implementation of inventory management}

The basic operations of inventory management include the operations of warehousing, inventory and monthly closing. When the materials are put in storage, the categories need to be selected, including purchase, the scarlet letter, inventory, adjustment and sales return. When the materials are delivery of cargo from storage, the categories also need to be selected, including picking, inventory, adjustment, the scarlet letter and scrap. And when the materials are inventory, the result is marked according to the difference between the inventory quantity and the actual quantity, and the inventory information is adjusted based on this.
The operations for the information of warehousing and inventory are similar, so the specific operation of warehouse receipt is illustrated as an example. When the receipt is added, the receipt number will be automatically generated based on the current time, which is unique and cannot be changed. The material information, including material name, category, supplier, specification, will be added to the receipt according the user's choice, then the quantity and price are added by user. After preserved, the total price of the warehouse receipt and the taxable price will be automatically calculated. After the addition is completed, the receipt can be submitted to the manager for review. At the same time, all warehousing information can be queried on the query interface.

The design of warehouse receipt maintenance interface is shown in Figure 4.

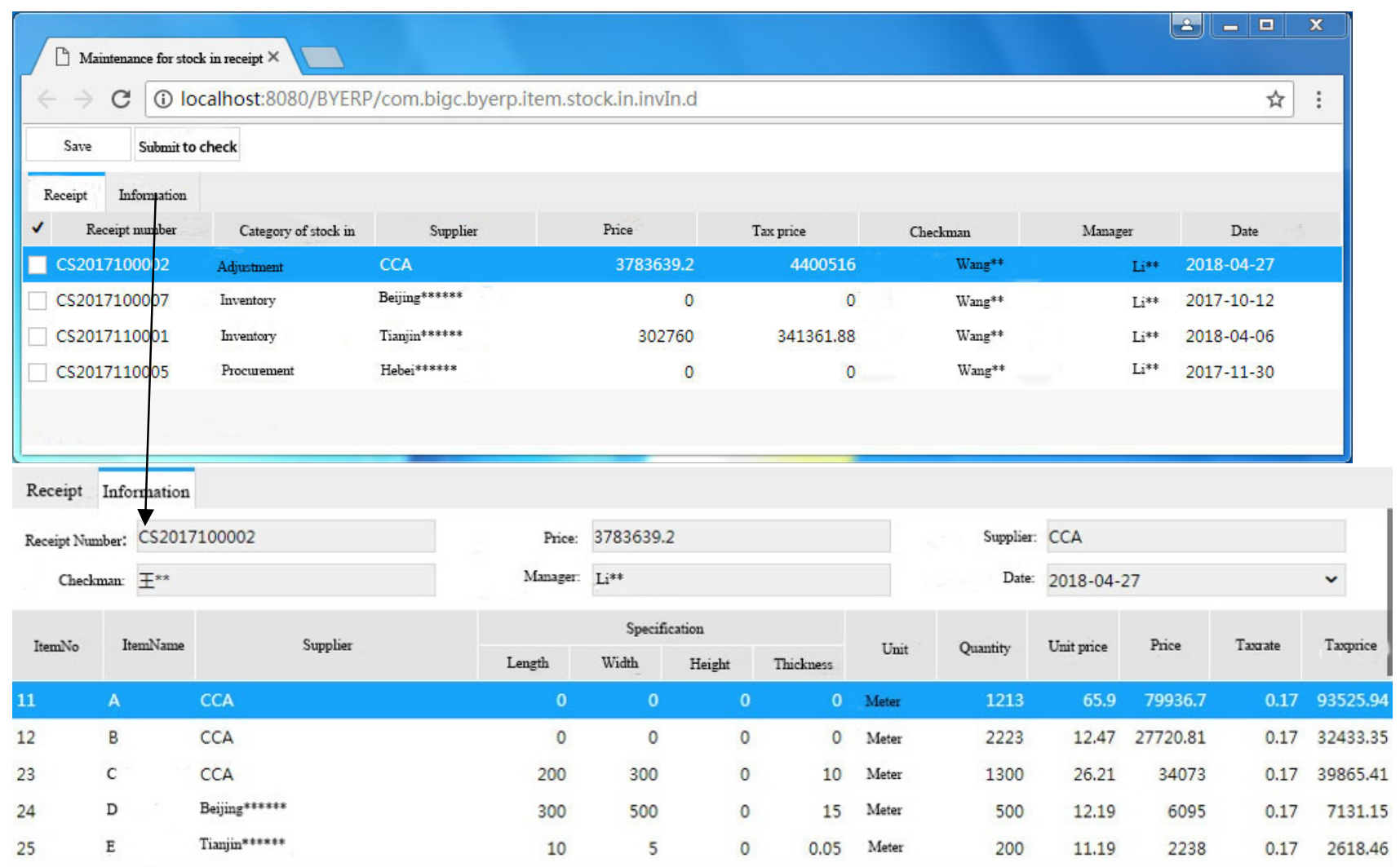

Figure 4. Design of warehouse receipt maintenance interface

Monthly closing refers to the summary of the information from all materials in and out of the previous month, including quantity and price. The specific process is as follows.

(1) The current date is obtained.

(2) Whether the month has been closed and whether there are any unchecked receipts.

(3) The first material information stored in stock table and the material information in the last month of the warehouse table according to the material number is obtained and calculated, then the first material information is obtained, the quantity and price are also summarized.
(4) The monthly closing table is traversed according to material number, and the balance information of the last month is obtained as the initial information.

(5) The balance information from the beginning information and the warehousing information of this monthly closing is calculated and obtained, which is added to the monthly closing table.

Also the entire stock table is traversed and the above process is repeated to get monthly closing information for all materials.

Throughout the business process, the timing diagram for the change of material information is shown in Figure 5. 


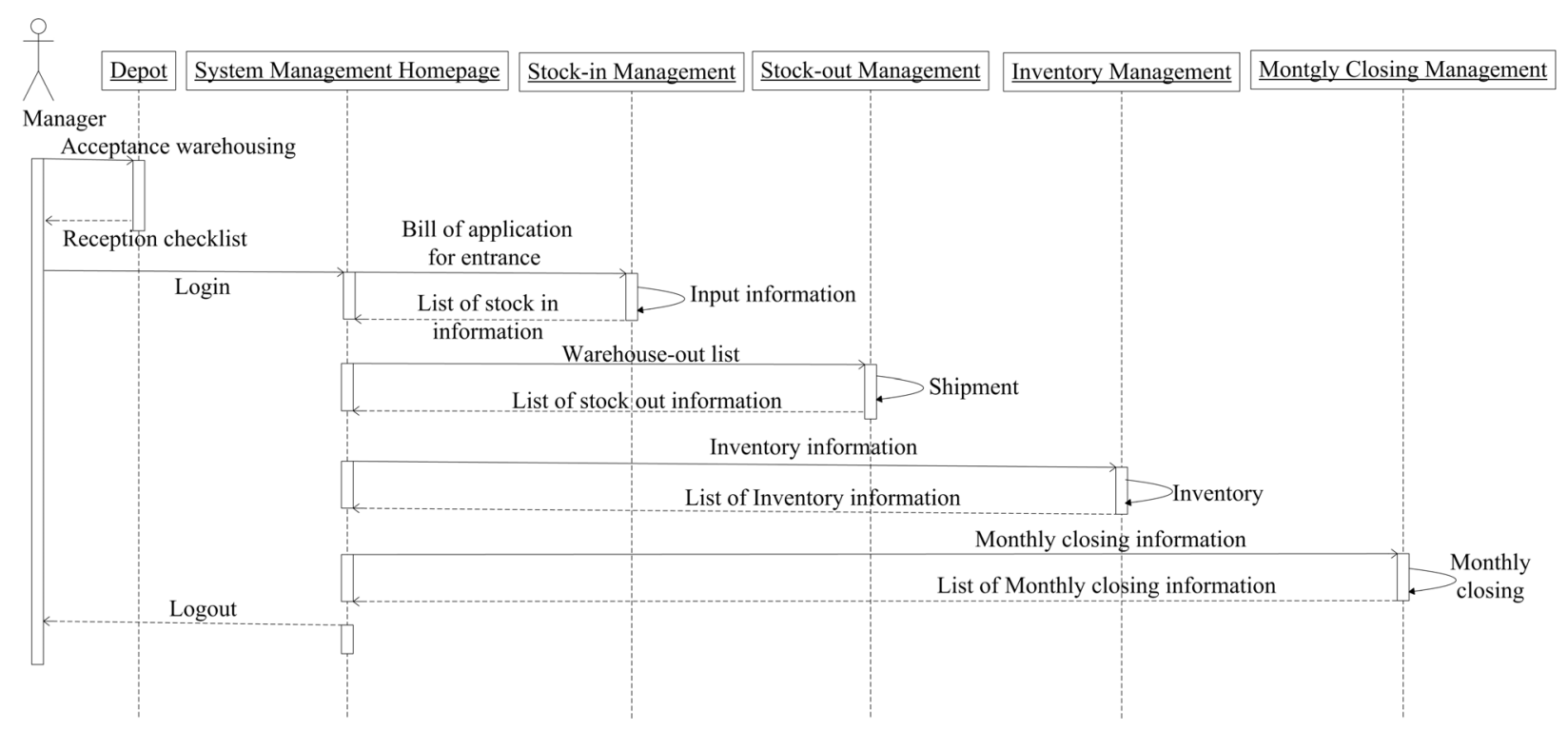

Figure 5. Design of timing diagram for the material information change

\section{Conclusion}

The business has been developed in the direction of many varieties and small batches in printing enterprise, and the inventory management needs to be more elaborate with the change in production methods. In order to solve the problems in traditional inventory management, the inventory management module is designed and implemented, which achieve the material warehousing, inventory, monthly closing functions. The interface is simple and easy to operate. It can accelerate the standardization operation and inquiry of material information. It changes the current situation of overloading work and material information storage in inventory management personnel. In the case of reducing the workload of the staff, it improves the level of enterprise information management and makes material management more orderly, efficient and accurate.

\section{Acknowledgment}

The paper is supported by National Natural Science "Nonlinear Dynamics model and Analysis of Gear Systems for Offset Press" (No.61472461).

\section{References}

1. Minghui Chai, Hongsen Yan, Peidi Qi. Design and Implementation of Material Management Information System Based on B/S [J].Computer Technology and Development, 2008, 1, 18(1).

2. Yuchun Gao, Yunping Sun. Development of Printing Enterprise Inventory Management System [J]. Market Modernization, 2012, 1.

3. Guanxia Shang. Enterprise Inventory Management Information System Design and Implementation [D]. Sichuan: University of Electronic Science and Technology, 2010.

4. Tingmei Zhou, Huijuan He, Yimin Mo. Research on Material Management Information System Based on B/S[J].Modern Manufacturing Engineering,2018(2).

5. Hanming Tang, Zhenxing Zhai, Baojun Guan, Xiao Huang. Easy to understand MySQL [M]. People's Posts and Telecommunications Press, 2008. 